\title{
On Dynamical Net-Charge Fluctuations within a Hadron Resonance Gas Approach
}

\author{
Abdel Nasser Tawfik, ${ }^{1,2}$ L. I. Abou-Salem, ${ }^{3}$ Asmaa G. Shalaby, ${ }^{3}$ and M. Hanafy ${ }^{3}$ \\ ${ }^{1}$ Egyptian Center for Theoretical Physics (ECTP), Modern University for Technology and Information (MTI), Cairo 11571, Egypt \\ ${ }^{2}$ World Laboratory for Cosmology and Particle Physics (WLCAPP), Cairo 11571, Egypt \\ ${ }^{3}$ Physics Department, Faculty of Science, Benha University, Benha 13518, Egypt \\ Correspondence should be addressed to Abdel Nasser Tawfik; a.tawfik@eng.mti.edu.eg
}

Received 12 January 2016; Revised 26 February 2016; Accepted 11 April 2016

Academic Editor: Aurelio Juste

Copyright (C) 2016 Abdel Nasser Tawfik et al. This is an open access article distributed under the Creative Commons Attribution License, which permits unrestricted use, distribution, and reproduction in any medium, provided the original work is properly cited. The publication of this article was funded by SCOAP ${ }^{3}$.

The dynamical net-charge fluctuations $\left(\nu_{\mathrm{dyn}}\right)$ in different particle ratios $K / \pi, K / p$, and $p / \pi$ are calculated from the hadron resonance gas (HRG) model and compared with STAR central Au+Au collisions at $\sqrt{s_{\mathrm{NN}}}=7.7-200 \mathrm{GeV}$ and NA49 central Pb+Pb collisions at $\sqrt{s_{\mathrm{NN}}}=6.3-17.3 \mathrm{GeV}$. The three charged particle ratios $(K / \pi, K / p$, and $p / \pi)$ are determined as total and average of opposite and average of the same charges. We find an excellent agreement between the HRG calculations and the experimental measurements, especially from STAR beam energy scan (BES) program, while the strange particles in the NA49 experiment at lower Super Proton Synchrotron (SPS) energies are not reproduced by the HRG approach. We conclude that the utilized HRG version seems to take into consideration various types of correlations including strong interactions through the heavy resonances and their decays especially at BES energies.

\section{Introduction}

The heavy-ion experiments are designed to study the nuclear matter under extreme conditions of high temperature or density or both of them [1]. A new state of matter called quark-gluon plasma (QGP), which is conjectured to shape the cosmic background geometry in a few microseconds after the Big Bang, is assumed to be created in heavy-ion collisions [2] and predicted by the quantum chromodynamics (QCD) [3]. The quark-hadron phase transition can be characterized by many experimental signatures [4-11] such as the netcharge fluctuations of various produced particles $[9,11-13]$.

The net-charge fluctuations can be measured [12-17] and calculated [8-10] in terms of different quantities such as the variance of the charge $V(Q)$, the variance of the charge ratios $V(R)$, the fluctuation of charged particle ratios $D$, and the dynamical net-charge fluctuations $v_{\mathrm{dyn}} . V(Q), V(R)$, and $D$ are affected by the measurement conditions such as the detector acceptance, the global charge conservation, the charge asymmetry, the background contributions, and the random efficiency loss $[9,18] \cdot v_{\text {dyn }}$ is the only quantity which can be measured independent of the detector acceptance; see, for instance, [9]. Furthermore, $v_{\mathrm{dyn}}$ is suitable for the calculations with antisymmetric charge distribution and the conservation of global charge [18].

For the sake of completeness, we mention that, in relativistic heavy-ion collisions, the event-by-event dynamical charge, the baryon number, and the strangeness fluctuations have been intensively investigated [7]. It was proposed that the dynamical charge fluctuations of positively to negatively charged pions give a measure for the number of rhoand omega-resonances which are likely produced through the hadronization process (quark-hadron phase transition) [7]. Therefore, the dynamical charge fluctuations have been proposed as a signature for the QGP formation [8] because the charge fluctuations are directly proportional to the square of the electric charge. In case of stable QGP, the charge fluctuations are slightly smaller than that in the hadron phase, due to the conservation of the charge fluctuations normalized to the entropy. Again, this signature was proposed because the fluctuations taking place in QGP are likely to be reduced through the rapid hadronization process $[8,19]$. Also, 
processes such as rescattering and resonance decay may weaken such fluctuations. The resonance decay which occurs after hadronization and stops at the stage of chemical freezeout should principally be distinguished from the resonance decay which shall be taken into consideration in the HRG approach. Furthermore, the dynamical charge fluctuations in QGP era are remarkably reduced from the ones characterizing the early stages of the heavy-ion collision which can be described by quarks and gluons degrees of freedom [20]. This illustrates the importance of considering the propagation of fluctuations from the very early stages of the collisions to the state of chemical freezeout via hadronization and other processes before drawing any conclusions about these fluctuations as a reflection of the QGP formation. The temporal evolution of this propagation can be evaluated by means of transport approaches, for instance. In the present work, we utilize a statistical approach, which characterizes very well the produced particles in their final state [21]. The studies of net-charge dynamical fluctuations, among others, are experimental tools to probe the final state of QCD processes.

In principle, the fluctuations can be estimated from statistical approaches as variance, covariance, or higher-order moment [21]. The dependence of mean transverse momentum and balance fluctuations on momentum is an essential tool to measure the fluctuations [22]. Any possible difference between the calculations and the measurements can be attributed to certain novel dynamics. These correlation functions are emerged as identical pion Hanbury BrownTwiss (HBT) correlations [23].

So far, the charge fluctuations have been analysed in several experiments. The PHENIX experiment has found that the charge fluctuations are consistent with that in hadron resonance gas (HRG) if the latter are extrapolated to larger detector acceptance, while the earlier are measured with a small rapidity acceptance [16]. The STAR experiment confirmed the PHENIX findings [6]. At SPS energies, both CERES [16] and NA49 [24] have reported consistency with pure pion gas predictions. Recently, the STAR experiment [25] measured the dependence of the dynamical net-charge fluctuations of $K / \pi, K / p$, and $p / \pi$ on the beam energy scan (BES) energies, $\sqrt{s_{\mathrm{NN}}}=7.7-200 \mathrm{GeV}$ [6]. The variance of the net-charge fluctuations has been estimated as a function of multiplicity, azimuthal coverage of the detector, and centrality [16] and are given as functions of the collision participants and the pseudorapidity, as well [12].

We present HRG calculations for the energy dependence of the dynamical net-charge fluctuations $\nu_{\text {dyn }}$ in different particle ratios: $K / \pi, p / \pi$, and $K / p$. We have considered three cases, total-charge ratios and average of the same and average of the opposite signs. We focus on $v_{\text {dyn }}$ as it is considered as a clean experimental signature not suffering from problems such as global charge conservation, charge asymmetry, background contributions, random efficiency loss, and volume fluctuations.

The present paper is organized as follows. The formalism of the HRG model is introduced in Section 2.1. Section 2.2 elaborates the net-charge fluctuations in particle ratios. The results and discussion of the net-charge fluctuations in $K / \pi$,
$K / p$, and $p / \pi$ are presented in Section 3. In Section 4 , the final conclusions are outlined.

\section{Formalism}

2.1. Hadron Resonance Gas Model. The thermodynamic properties can be directly derived from the partition function $Z(\beta, \mu, V)$. In a grand canonical ensemble [26]

$$
Z(\beta, \mu, V)=\operatorname{Tr}\{\exp [(\mu N-H) \beta]\},
$$

where $\beta=1 / T$ is the inverse of the temperature $(T), H$ represents the Hamiltonian of the system, $N$ is the number of particles, $V$ is the volume of the interacting system, and $\mu$ is the chemical potential. The Hamiltonian is included as it contains all relevant degrees of freedom of the confined and the strongly interacting medium and implicitly includes other types of interactions which result in the resonances formation [26-30]. The grand canonical ensemble has two important features: the kinetic energies and the summation over all degrees of freedom and energies of the hadron resonances. As Hagedorn proposed, the formation of resonances can only be achieved through strong interactions, that is, "resonances (fireballs) are composed of further resonances (fireballs), which in turn consist of resonances (fireballs) and so on." This simply means that the contributions of the various hadron resonances to the total partition function are the same as those of ideal collisionless constituents with an effective mass. At a temperature compatible with the resonance half-width, the effective mass approaches the physical one [3]. This implies that at high temperatures even strong interactions are conjectured to be taken into consideration through including heavy resonances.

Therefore, the partition function, (1), of a hadron resonance gas can be summed over the number of the hadron resonances composing the HRG degrees of freedom [31]

$$
\ln Z(\beta, \mu, V)=\sum_{i} \ln Z_{i}(\beta, \mu, V)
$$

where $\mu \in\left[\mu_{B}, \mu_{S}, \mu_{Q}\right]$ are the chemical potentials related to the baryon number, the strangeness, and the electric charge, respectively. The chemical potentials $(\mu)$ can be related to the nucleus-nucleus center-of-mass energy $\left(\sqrt{s_{\mathrm{NN}}}\right)$, phenomenologically [21, 32, 33],

$$
\mu=\frac{a}{1+b \sqrt{s_{\mathrm{NN}}}},
$$

where $a=1.245 \pm 0.094 \mathrm{GeV}$ and $b=0.264 \pm 0.028 \mathrm{GeV}^{-1}$.

It should be highlighted that the hadron resonances with masses up to $2 \mathrm{GeV}$ include a suitable set of constituents needed for the partition function. The concrete number of constituents does not matter. Nevertheless, this can be determined from the latest update (2014) of the particle data group. We have included all mesons and baryons having masses $\leq 2 \mathrm{GeV}$. Such a mass cut is defined in order to avoid the singularity expected at the Hagedorn temperature [2629]. The strong interactions are assumed to be taken into consideration. In light of this, the validity of HRG is limited to temperatures below the critical one, $T_{c}$. 
In high energy experiments, the produced particles and their correlations and fluctuations are believed to provide essential information about the nature, size, and composition of the QCD matter from which they are stemming. The freezeout parameters have been determined at various centerof-mass energies $\sqrt{s_{\mathrm{NN}}}$. These are temperature $T$ and baryon chemical potential $\mu$. The chemical freezeout is a stage in which the inelastic collisions entirely cease and the relative particle yields and ratios get fixed. As given in (3), $\mu$ is related to $\sqrt{s_{\mathrm{NN}}}$ (also $T$ can be given in dependence on $\sqrt{s_{\mathrm{NN}}}$ ). $T$ versus $\mu$ has been phenomenologically described, the socalled freezeout conditions, for instance, $s / T^{3}=7$ [21]. Accordingly, temperatures and baryon chemical potentials can be determined for each beam energy, for instance, (3). Further interrelations can be taken from [21, 32, 33].

The $i$ th hadron (resonance) partition function can be written as

$$
\begin{aligned}
& \ln Z_{i}(\beta, \mu, V) \\
& = \pm \frac{V g_{i}}{2 \pi^{2}} \int_{0}^{\infty} k^{2} d k \ln \left[1 \pm \lambda_{i} \exp \left(-\beta \varepsilon_{i}(k)\right)\right]
\end{aligned}
$$

where \pm stand for fermions and bosons, respectively, $\varepsilon_{i}(k)=$ $\sqrt{k^{2}+m_{i}^{2}}$, and the fugacity factor $\lambda_{i}$ [28] is given by

$$
\lambda_{i}(\beta, \mu)=\exp \left[\left(B_{i} \mu_{B}+S_{i} \mu_{S}+Q_{i} \mu_{\mathrm{Q}}\right) \beta\right]
$$

where $B_{i}, S_{i}$, and $Q_{i}$ are baryon, strange, and charge quantum number of $i$ th hadron resonance.

The number density can be obtained as [31]

$$
n(\beta, \mu)=\sum_{i} \pm \frac{g_{i}}{2 \pi^{2}} \int_{0}^{\infty} \frac{k^{2} d k}{\exp \left[\left(\mu_{i}-\varepsilon_{i}(k)\right) \beta\right] \pm 1} .
$$

In Section 2.2, the dynamical net-charge fluctuations shall be derived in the HRG approach.

In determining $n(\beta, \mu)$ as an example on all other thermodynamic quantities, we take into account contributions from possible decay channels. Thus for $m$ th species, the final particle number density is calculated as

$$
n_{m}^{\text {total }}(\beta, \mu)=n_{m}^{\text {direct }}(\beta, \mu)+\sum_{n \neq m} B_{n \rightarrow m} n_{n}^{\text {decaying }}(\beta, \mu),
$$

where $B_{n \rightarrow m}$ is the decay branching ratio of $n$th into $m$ th hadron resonance and $n_{n}^{\text {decaying }}$ is the number density of $n$th resonance that decays in the particle species of interest.

2.2. Dynamical Net-Charge Fluctuations. Predicting the phase transition can be achieved through different signatures such as the fluctuations of the net-charges of the produced particles. As discussed in previous sections, the net-charge fluctuations as a signature for QGP formation is best accessible through $\nu_{\text {dyn }}[9,18]$.
The standard deviation which describes the fluctuations in $p / \pi$ ratio, for instance, is given as [34-36]

$$
\begin{aligned}
\sigma_{p / \pi}^{2}= & \frac{\left\langle N_{p}{ }^{2}\right\rangle-\left\langle N_{p}\right\rangle^{2}}{\left\langle N_{p}\right\rangle^{2}}+\frac{\left\langle N_{\pi}^{2}\right\rangle-\left\langle N_{\pi}\right\rangle^{2}}{\left\langle N_{\pi}\right\rangle^{2}} \\
& -2 \frac{\left\langle N_{p} N_{\pi}\right\rangle-\left\langle N_{p}\right\rangle\left\langle N_{\pi}\right\rangle}{\left\langle N_{p}\right\rangle\left\langle N_{\pi}\right\rangle},
\end{aligned}
$$

where $N_{p}$ and $N_{\pi}$ represent the number of charged protons and pions, respectively. The covariance measures how much two random variables change with each other. The variance is a special case of covariance if the variables are identical. The quantity $\left\langle N_{p} N_{\pi}\right\rangle$ gives the statistical average of the integrals or multiplicities of the simultaneous correlation of the production of both $p$ and $\pi$.

Equation (8) can be rewritten as

$$
\begin{aligned}
\sigma_{p / \pi}^{2}= & \frac{\left\langle N_{p}\left(N_{p}-1\right)\right\rangle}{\left\langle N_{p}\right\rangle^{2}}+\frac{\left\langle N_{\pi}\left(N_{\pi}-1\right)\right\rangle}{\left\langle N_{\pi}\right\rangle^{2}} \\
& -2 \frac{\left\langle N_{p} N_{\pi}\right\rangle}{\left\langle N_{p}\right\rangle\left\langle N_{\pi}\right\rangle}+\frac{1}{\left\langle N_{p}\right\rangle}+\frac{1}{\left\langle N_{\pi}\right\rangle} .
\end{aligned}
$$

The last two terms in (9) represent static net-charge fluctuations in Poisson's limit (uncorrelated particle production, i.e., just statistical). Then, the charge dependence of the dynamical net-charge fluctuations for $p / \pi$ charge ratio can be given in terms of second-order factorial moments [3739], $\left\langle N_{p}\left(N_{p}-1\right)\right\rangle$ and/or $\left\langle N_{\pi}\left(N_{\pi}-1\right)\right\rangle$, and the covariance $\left\langle N_{p} N_{\pi}\right\rangle$,

$$
\begin{aligned}
\left.\nu_{\text {dyn }}\right|_{p / \pi}= & \frac{\left\langle N_{p}\left(N_{p}-1\right)\right\rangle}{\left\langle N_{p}\right\rangle^{2}}+\frac{\left\langle N_{\pi}\left(N_{\pi}-1\right)\right\rangle}{\left\langle N_{\pi}\right\rangle^{2}} \\
& -2 \frac{\left\langle N_{p} N_{\pi}\right\rangle}{\left\langle N_{p}\right\rangle\left\langle N_{\pi}\right\rangle} .
\end{aligned}
$$

The dynamical fluctuations are related to single- and twoparticle multiplicities. Straightforwardly, the expressions for $K / \pi$ and $K / p$ ratios can be derived as in (10). For more information about the net-charge fluctuations, the readers are kindly advised to consult [40-43].

The net-charge dynamical fluctuations $(\nu)$ may be affected by uncertainties arising from volume fluctuations and thus the study of particle (charge) ratio fluctuations is assumed to largely eliminate these uncertainties. $v$ might be influenced by the charge conservation and simultaneously by the limited charge available on the onset on the collision [9]. The study of particle (charge) ratio $v$ gives a measure for the relative correlation strength of particle pairs. Negative $v$ signifies a dominant contribution from correlated particle pairs.

In the statistical thermal model, the main source of correlations is the resonance decay; that is, the second term in (7) is the only source of different-species correlations. 


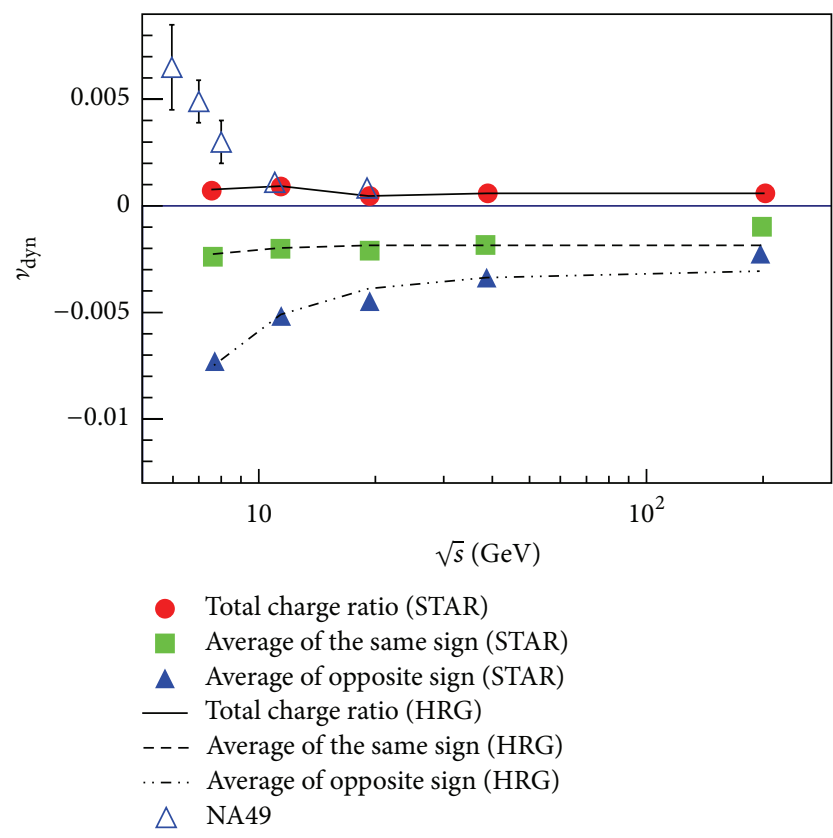

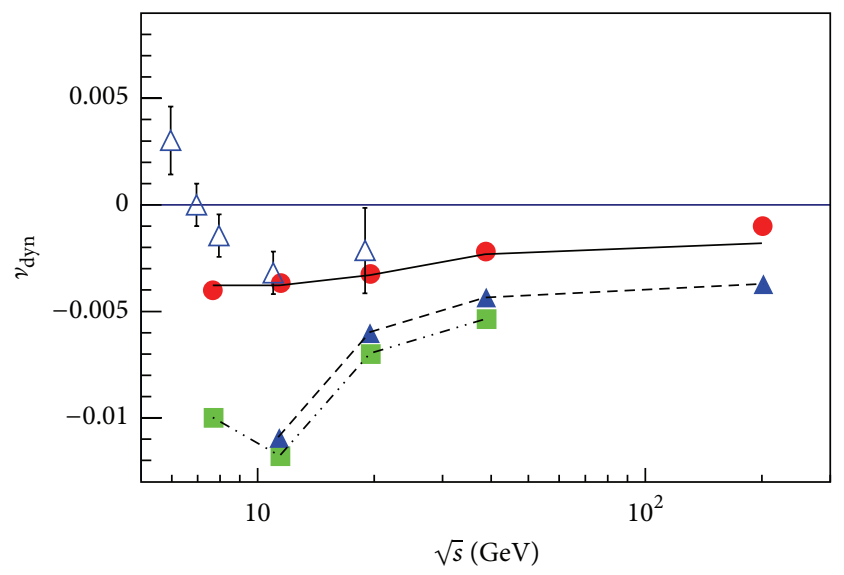

- Total charge ratio (STAR)

- Average of the same sign (STAR)

- Average of opposite sign (STAR)

- Total charge ratio (HRG)

- - - Average of the same sign (HRG)

- Average of opposite sign (HRG)

$\triangle \quad$ NA49

(a)

(b)

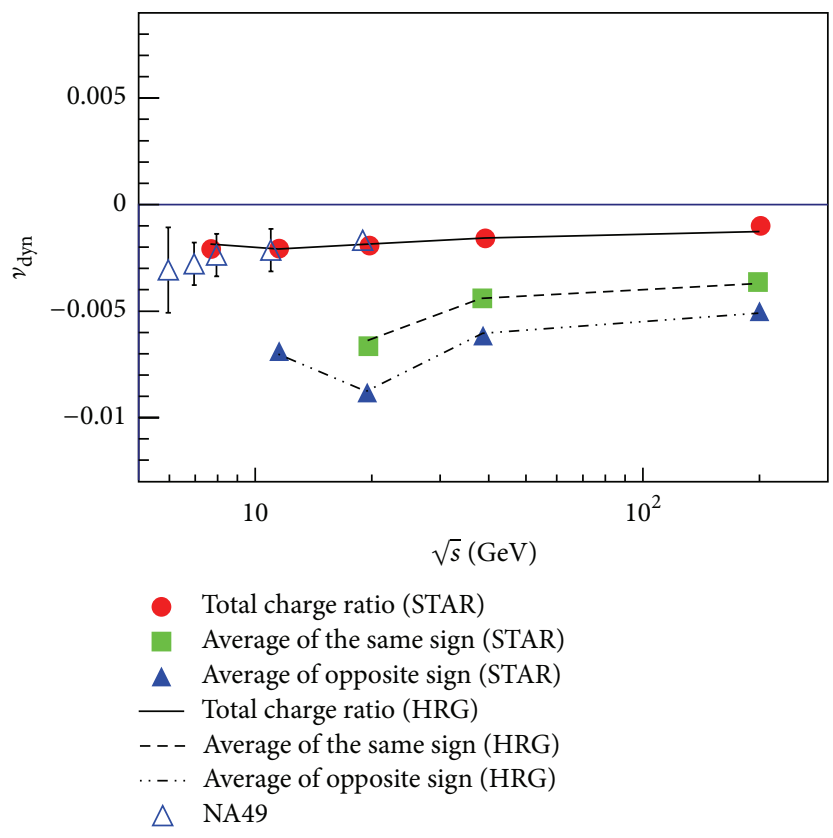

(c)

FIGURE 1: The energy dependence of the dynamical net-charge fluctuations $v_{\text {dyn }}$ calculated from the HRG model is compared with STAR [25] (solid symbols) and NA49 [34] (open triangles) measurements for the charge ratios $K / \pi(\mathrm{a}), K / p$ (b), and $p / \pi$ (c). The fluctuations of the charge ratios are determined as total and average of the same and average of opposite charge-signs.

For instance, $\Delta$ baryon decaying into a nucleon and a pion certainly results in proton-pion correlation. Similarly, the difference between $\left\langle N_{p} N_{K}\right\rangle$ and $\left\langle N_{p}\right\rangle\left\langle N_{K}\right\rangle$ must come from a strange baryon decay.

\section{Results and Discussion}

In Figure 1, the dynamical net-charge fluctuations calculated from the HRG model are given as a function of beam energy for various charge ratios $K / \pi, K / p$, and $p / \pi$. The HRG calculations are compared with STAR [25] (solid symbols) and NA49 [34] (open triangles) measurements. For each charge ratio, three different cases are considered, namely, the total dynamical fluctuations and the average of the same and of opposite charge-signs, which are characterized by solid, dashed, double-dot-dashed curves, respectively. The charge dependence of the dynamical net-charge fluctuations is conjectured to give detailed information about the correlations 
of various produced particles (charges) and on how they are correlated with each other.

Figure 1(a) shows $\nu_{\text {dyn }}$ for $K / \pi$ charge ratio as a function of the beam energy. The HRG calculations are compared with STAR measurement in central $0-5 \% \mathrm{Au}+\mathrm{Au}$ collisions at $\sqrt{s_{\mathrm{NN}}}=7.7-200 \mathrm{GeV}[25]$ and NA49 for total charged particle ratios in central $0-3.5 \% \mathrm{~Pb}+\mathrm{Pb}$ collisions at $\sqrt{s_{\mathrm{NN}}}=$ 6.3-17.3 GeV [34]. The three cases, namely, dynamical fluctuations for total charges $\left(K^{+}+K^{-}\right) /\left(\pi^{+}+\pi^{-}\right)$, average of the same charges $K^{+} / \pi^{+}+K^{-} / \pi^{-}$, and average of opposite signs $K^{+} / \pi^{-}+K^{-} / \pi^{+}$are compared with each other. Only $\left(K^{+}+K^{-}\right) /\left(\pi^{+}+\pi^{-}\right)$charge ratios have positive dynamical fluctuations, which remain constant with increasing the beam energy. Almost the same dependence is also observed in the average of the same charges but with negative dynamical netcharge fluctuations. The average of opposite charges has a stronger energy dependence. It is obvious that the dynamical fluctuations of opposite-charged ratios have larger negative values, which decline with increasing of the collision energy, than that of the same-charged ratios. The negative values for the dynamical net-charge fluctuations can be understood from (10). The last term (the correlation term) seems to have a larger value than the first two terms. Thus, the largest negative values obtained in opposite-charged particle ratios mean a remarkably larger cross-correlation of these two charges.

Figure 1(b) presents the same as in Figure 1(a) but for $K / p$ charge ratio. The dynamical fluctuations for total charges increase and then saturate with increasing $\sqrt{s_{\mathrm{NN}}}$. The dynamical fluctuations of average of the same-charged particles obviously increase with the energy. For oppositely charged particles ratios the dynamical fluctuations first decrease and then increase with increasing of the energy. Such a remarkable dependence needs a further confirmation at lower energies. Unfortunately, no experimental measurements are available so far. This might be possible with the NuclotronBased Ion Collider Facility (NICA) future facility at the Joint Institute for Nuclear Research (JINR) at Dubna, Russia, and the Facility for Antiproton and Ion Research (FAIR) at the Gesellschaft für Schwerionenforschung (GSI) at Darmstadt, Germany. We observe that the values of the dynamical fluctuations of $K / p$ are always negative. This means that the produced kaons and protons are strongly correlated over all beam energies.

Figure 1(c) depicts the same as in previous panels but for $p / \pi$ charge ratios. Their total-charge dynamical fluctuations slightly increase with the energy. Oppositely charged particles ratios first decrease and then increase with the energy. Again, further experimental measurements at lower energies shall play a crucial role in confirming this remarkable dependence. The dynamical fluctuations of the same-signed $p / \pi$ increase with the energy. As in $K / p$ charged particles ratios, here the dynamical fluctuations are always negative.

We have observed that the dynamical net-charge fluctuations possess a remarkable dependence on the beam energy, from which we follow the change in their values and thus might be able to signal the impact of QGP formation. The excellent agreement between the HRG calculations and the STAR measurements gives indication for the statistical origin of the particle production (charges). The excellent agreement reveals that the HRG fluctuations reproduce the ones for the produced particles which are conjectured to undergo a quarkhadron phase transition. The phase transition itself is not accessible by the HRG model due to absence of the underlying dynamics and missing of the degrees of freedom at least of the QGP phase. On the other hand, the produced particles in their final state (chemical freezeout) are likely affected by the QCD phase transition(s). For the sake of completeness, the version of the HRG model we have utilized in the present work [21] is conjectured to take into consideration various types of correlations including the strong interactions through the summation over heavy resonances [3]. While the HRG calculations are excellently consistent with the results from STAR experiment, they are not compatible with the NA49 measurements, especially for strange particles at lower SPS energies. Besides strangeness production at low energies, the Relativistic Heavy-Ion Collider (RHIC) experiments are well suited for weak decay contributions and operate various specialized detectors which is not the case for the SPS experiments [44].

\section{Conclusions}

In the present work, the dynamical net-charge fluctuations which are proposed as signatures of the QGP formation are calculated from $v_{\mathrm{dyn}}$ by using the HRG approach, in which the entire list of baryons and mesons with masses $\leq 2 \mathrm{GeV}$ from the latest particle data group is taken into account. This includes stable hadrons and unstable resonances. The latter can be decaying into the particles (charges) of interest and therefore their branching ratios are taken into consideration. For the sake of completeness, we emphasize that the constituents of the HRG models are assumed as point-like particles, that is, no excluded-volume corrections were added. $v_{\text {dyn }}$ is considered as a suitable measurable quantity for the dynamical net-charge fluctuations. We have calculated the charge dependence of the dynamical net-charge fluctuations in $K / \pi, K / p$, and $p / \pi$ charge ratios and compared the results with the STAR and NA49 corresponding measurements. An excellent agreement with the STAR BES results for the three charge ratios at $\sqrt{s_{\mathrm{NN}}}=7.7-200 \mathrm{GeV}$ is obtained. On the other hand, HRG agrees well with NA49 results at top SPS energies. But at lower SPS energies, HRG is not capable of reproducing NA49 dynamical net-charge fluctuations for strange particles, particularly. We found that only $\left(K^{+}+\right.$ $\left.K^{-}\right) /\left(\pi^{+}+\pi^{-}\right)$are positive. All other charge ratios and combinations of various charges result in negative net-charge fluctuations indicating that the cross term, last term in (10), the correlated particle pair, is dominant against the first two terms (single uncorrelated particle). Thus, we conclude that the correlations between charge ratios are larger than the dynamical fluctuations in the noncorrelated charges.

To the authors' knowledge this is the first time in which the HRG approach is implemented in calculating the dynamical net-charge fluctuations $\left(v_{\text {dyn }}\right)$ for different charge ratios as a function of energy and compared with STAR and NA49 experiments. We conclude that, at least, the HRG version utilized in the present work (all particle data group's baryons and baryons with masses $\leq 2 \mathrm{GeV}$ and possible branching 
ratios (decay channels in the particles or charges of interest) but no excluded-volume corrections) seems to count for various types of correlations including the strong interactions obviously through the sum over heavy resonances, especially at BES energies.

\section{Competing Interests}

The authors declare that there is no conflict of interests regarding the publication of this paper.

\section{References}

[1] N. Cabibbo and G. Parisi, "Exponential hadronic spectrum and quark liberation," Physics Letters B, vol. 59, no. 1, pp. 67-69, 1975.

[2] M. Gyulassy and L. McLerran, "New forms of QCD matter discovered at RHIC," Nuclear Physics A, vol. 750, no. 1, pp. 3063, 2005.

[3] A. Tawfik, "QCD phase diagram: a comparison of lattice and hadron resonance gas model calculations," Physical Review D, vol. 71, no. 5, Article ID 054502, 11 pages, 2005.

[4] T. J. Tarnowsky, "Charge dependence and scaling properties of dynamical $K / \pi, p / \pi$, and $K / p$ fluctuations from the STAR experiment," Acta Physica Polonica B, Proceedings Supplement, vol. 5, p. 515, 2012.

[5] B. I. Abelev, M. Aggarwal, Z. Ahammed et al., "K/pi fluctuations at relativistic energies," Physical Review Letters, vol. 103, no. 9, Article ID 092301, 2009.

[6] J. Adams, M. M. Aggarwal, Z. Ahammed et al., "Multiplicity fluctuations in Au+Au collisions at $s_{N N}{ }^{1 / 2}=130 \mathrm{GeV}$," Physical Review C, vol. 68, Article ID 044905, 2003.

[7] S. Jeon and V. Koch, "Fluctuations of particle ratios and the abundance of hadronic resonances," Physical Review Letters, vol. 83, article 5435, 1999.

[8] S. Jeon and V. Koch, "Charged particle ratio fluctuation as a signal for quark-gluon plasma," Physical Review Letters, vol. 85, no. 10, pp. 2076-2079, 2000.

[9] C. Pruneau, S. Gavin, and S. Voloshin, "Methods for the study of particle production fluctuations," Physical Review C, vol. 66, no. 4, Article ID 044904, 12 pages, 2002.

[10] D. Bower and S. Gavin, "Baryon fluctuations and the QCD phase transition," Physical Review C-Nuclear Physics, vol. 64, no. 5, pp. 519021-519025, 2001.

[11] C. A. Pruneau and The STAR Collaboration, "Event by event net charge fluctuations," Heavy Ion Physics, vol. 21, no. 2, pp. 261-266, 2004, http://link.springer.com/article/ 10.1556\%2FAPH.21.2004.2-4.25.

[12] B. Abelev, J. Adam, D. Adamová et al., "Net-charge fluctuations in $\mathrm{Pb}-\mathrm{Pb}$ collisions at $\sqrt{s_{\mathrm{NN}}}=2.76 \mathrm{TeV}$," Physical Review Letters, vol. 110, no. 15, Article ID 152301, 2013.

[13] M. Weber, "Net-charge fluctuations and balance functions at the LHC," Nuclear Physics A, vol. 904-905, pp. 467c-470c, 2013.

[14] M. Weber, "Correlations and fluctuations studied with ALICE," Journal of Physics: Conference Series, vol. 389, no. 1, Article ID 012036, 2012.

[15] Y. Zhou, K.-J. Wu, and F. Liu, "Charged particle fluctuation in $\mathrm{Au}+\mathrm{Au}$ collision," Chinese Physics C, vol. 34, no. 9, article 1436, 2010.

[16] K. Adcox, S. S. Adler, N. N. Ajitanand et al., "Net charge fluctuations in $\mathrm{Au}+\mathrm{Au}$ interactions at $\sqrt{s_{N N}}=130 \mathrm{GeV}$," Physical Review Letters, vol. 89, no. 8, Article ID 082301, 2002.
[17] B. I. Abelev, M. M. Aggarwal, Z. Ahammed et al., "Beamenergy and system-size dependence of dynamical net charge fluctuations," Physical Review C, vol. 79, no. 2, Article ID 024906, 2009.

[18] P. Christiansen, E. Haslum, and E. Stenlund, "Number-ratio fluctuations in high-energy particle production," Physical Review C, vol. 80, no. 3, Article ID 034903, 2009.

[19] V. Koch, M. Bleicher, and S. Jeon, "Event-by-event fluctuations and the QGP," Nuclear Physics A, vol. 698, no. 1-4, pp. 261-268, 2002.

[20] T. K. Nayak, "Overview of event-by-event analysis of high energy nuclear collisions," International Journal of Modern Physics E, vol. 16, no. 10, pp. 3303-3322, 2007.

[21] A. N. Tawfik, "Equilibrium statistical-thermal models in highenergy," International Journal of Modern Physics A, vol. 29, Article ID 1430021, 2014.

[22] A. Tawfik and A. G. Shalaby, "Balance function in high-energy collisions," Advances in High Energy Physics, vol. 2015, Article ID 186812, 24 pages, 2015.

[23] G. A. Miller and J. G. Cramer, "Nucleus-nucleus scattering at relativistic energies," Nuclear Physics A, vol. 782, pp. 251-258, 2007.

[24] C. Blume, T. Anticic, B. Baatar et al., "Results on correlations and fluctuations from NA49," Nuclear Physics A, vol. 715, pp. $55 c-64 c, 2003$.

[25] T. J. Tarnowsky, B. K. Srivastava, P. P. Scharenberg et al., "Charge dependence and scaling properties of dynamical $K / \pi, p / \pi$, and $K / p$ fluctuations from the STAR experiment," Acta Physica Polonica B, Proceedings Supplement, vol. 5, p. 515, 2012.

[26] F. Karsch, K. Redlich, and A. Tawfik, "Hadron resonance mass spectrum and lattice QCD thermodynamics," The European Physical Journal C, vol. 29, no. 4, pp. 549-556, 2003.

[27] F. Karsch, K. Redlich, and A. Tawfik, "Thermodynamics at non-zero baryon number density: a comparison of lattice and hadron resonance gas model calculations," Physics Letters B, vol. 571, no. 1-2, pp. 67-74, 2003.

[28] K. Redlich, F. Karsch, and A. Tawfik, "Heavy-ion collisions and lattice QCD at finite baryon density," Journal of Physics G: Nuclear and Particle Physics, vol. 30, no. 8, pp. S1271-S1274, 2004.

[29] A. Tawfik, "The influence of strange quarks on QCD phase diagram and chemical freeze-out: results from the hadron resonance gas model," Journal of Physics G, vol. 31, pp. S1105S1110, 2005.

[30] R. Venugopalan and M. Prakash, "Thermal properties of interacting hadrons," Nuclear Physics A, vol. 546, no. 4, pp. 718-760, 1992.

[31] A. Tawfik, "Antiproton-to-proton ratios for ALICE heavy-ion collisions," Nuclear Physics A, vol. 859, no. 1, pp. 63-72, 2011.

[32] A. N. Tawfik and E. Abbas, "Thermal description of particle production in Au-Au collisions at STAR energies," Physics of Particles and Nuclei Letters, vol. 12, no. 4, pp. 521-531, 2015.

[33] A. N. Tawfik, M. Y. El-Bakry, D. M. Habashy, M. T. Mohamed, and E. Abbas, "Degree of chemical non-equilibrium in central $\mathrm{Au}-\mathrm{Au}$ collisions at RHIC energies," International Journal of Modern Physics E, vol. 24, Article ID 1550067, 2015.

[34] C. Alt, T. Anticic, B. Baatar et al., "Energy dependence of particle ratio fluctuations in central $\mathrm{Pb}+\mathrm{Pb}$ collisions from $\sqrt{s_{N N}}=6.3$ to 17.3 GeV," Physical Review C, vol. 79, no. 4, Article ID 044910, 2009. 
[35] T. Anticic, B. Baatar, D. Barna et al., "Energy dependence of kaon-to-proton ratio fluctuations in central $\mathrm{Pb}+\mathrm{Pb}$ collisions from $\sqrt{s_{\mathrm{NN}}}=6.3$ to $17.3 \mathrm{GeV}$,' Physical Review $C$, vol. 83, Article ID $061902(\mathrm{R}), 2011$.

[36] S. Jeon and V. Koch, Quark Gluon Plasma 3, Edited by R. C. Hwa and X.-N. Wang, World Scientific, Singapore, 2004.

[37] M. I. Adamovich, M. M. Aggarwal, Y. A. Alexandrov et al., "Nuclear effect in higher-dimensional factorial moment analysis of the ${ }^{16} \mathrm{O}-,{ }^{32} \mathrm{~S}$ - and ${ }^{197} \mathrm{Au}-\mathrm{Em}$ interaction data at 200, 60 and $11 \mathrm{~A} \mathrm{GeV/c,"} \mathrm{Zeitschrift} \mathrm{für} \mathrm{Physik} \mathrm{C} \mathrm{Particles} \mathrm{and} \mathrm{Fields,} \mathrm{vol.}$ 76, no. 4, pp. 659-663, 1997.

[38] M. I. Adamovich, M. M. Aggarwal, N. P. Andreeva et al., "Factorial moments of Si-28 induced interactions with $\mathrm{Ag}(\mathrm{Br})$ nuclei," Heavy Ion Physics, vol. 13, no. 4, pp. 213-221, 2001.

[39] A. M. Tawfik, "Factorial moments of the multiplicity distribution in $\mathrm{Pb}+\mathrm{Pb}$ collisions at $158-\mathrm{A}-\mathrm{GeV}$," Journal of Physics $\mathrm{G}$ : Nuclear and Particle Physics, vol. 27, no. 11, p. 2283, 2001.

[40] V. Koch, Relativistic Heavy Ion Physics, Edited by R. Stock, Springer, Heidelberg, Germany, 2010.

[41] A. Bialas and V. Koch, "Event-by-event fluctuations and inclusive distributions," Physics Letters, Section B: Nuclear, Elementary Particle and High-Energy Physics, vol. 456, no. 1, pp. 1-4, 1999.

[42] A. Bialas, "Charge fluctuations in a quark-antiquark system," Physics Letters B, vol. 532, no. 3-4, pp. 249-251, 2002.

[43] H. Heiselberg and A. D. Jackson, "Anomalous multiplicity fluctuations from phase transitions in heavy ion collisions," Physical Review C, vol. 63, Article ID 064904, 2001.

[44] A. Andronic, P. Braun-Munzinger, and J. Stachel, "Hadron production in central nucleus-nucleus collisions at chemical freezeout," Nuclear Physics A, vol. 772, no. 3-4, pp. 167-199, 2006. 

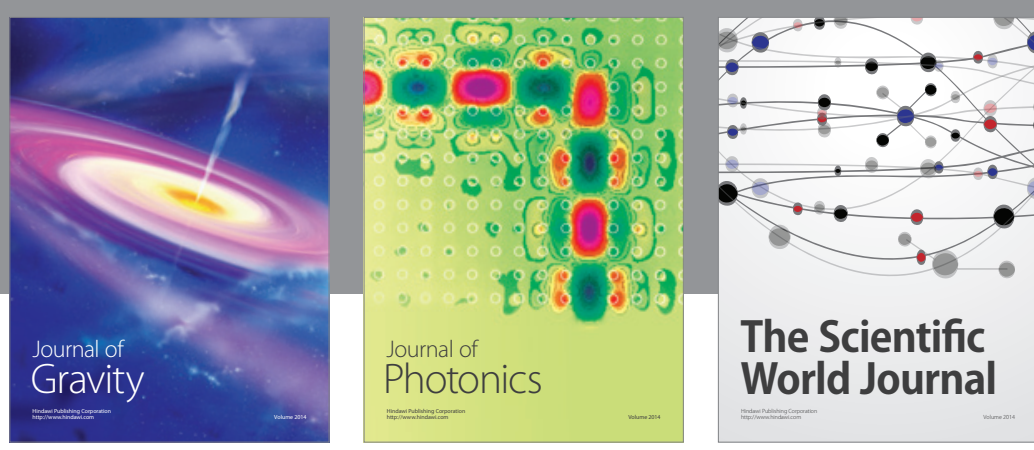

The Scientific World Journal
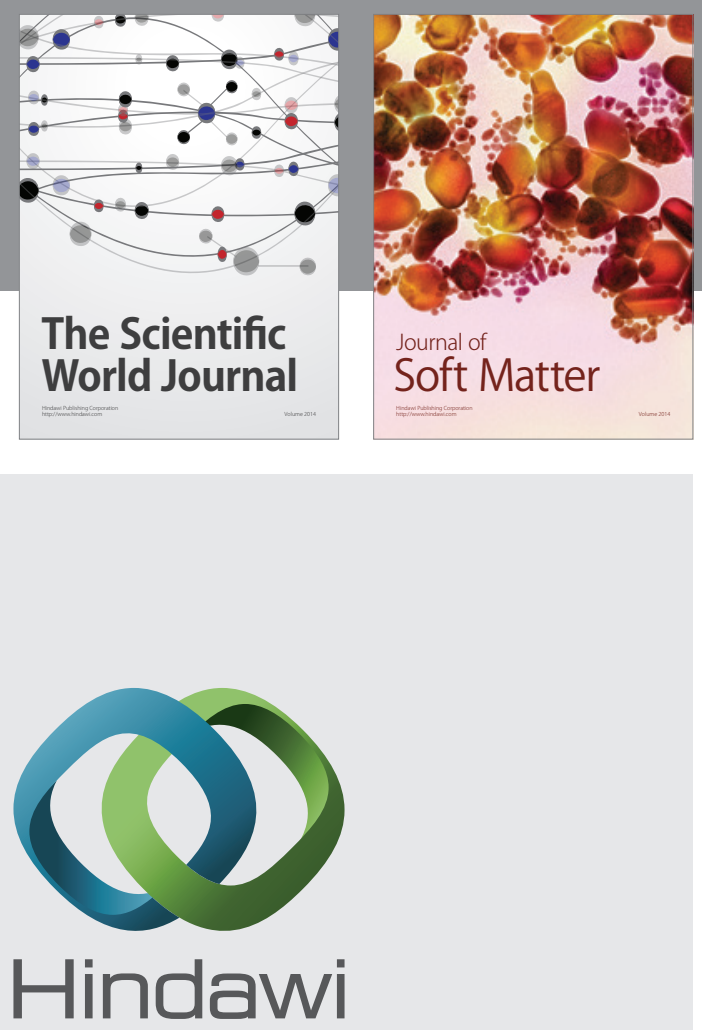

Submit your manuscripts at

http://www.hindawi.com

nternational Journal of

Statistical Mechanics
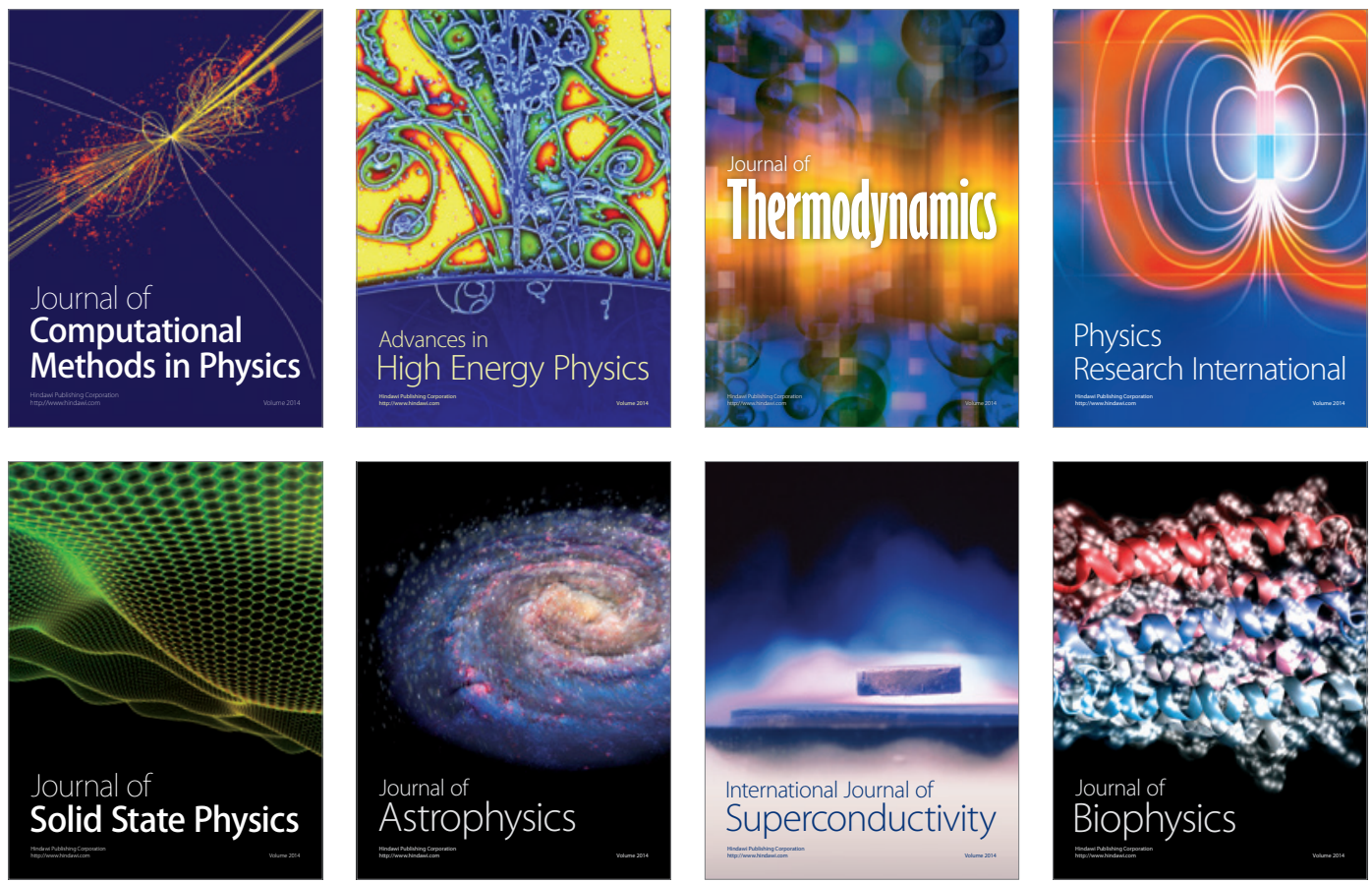
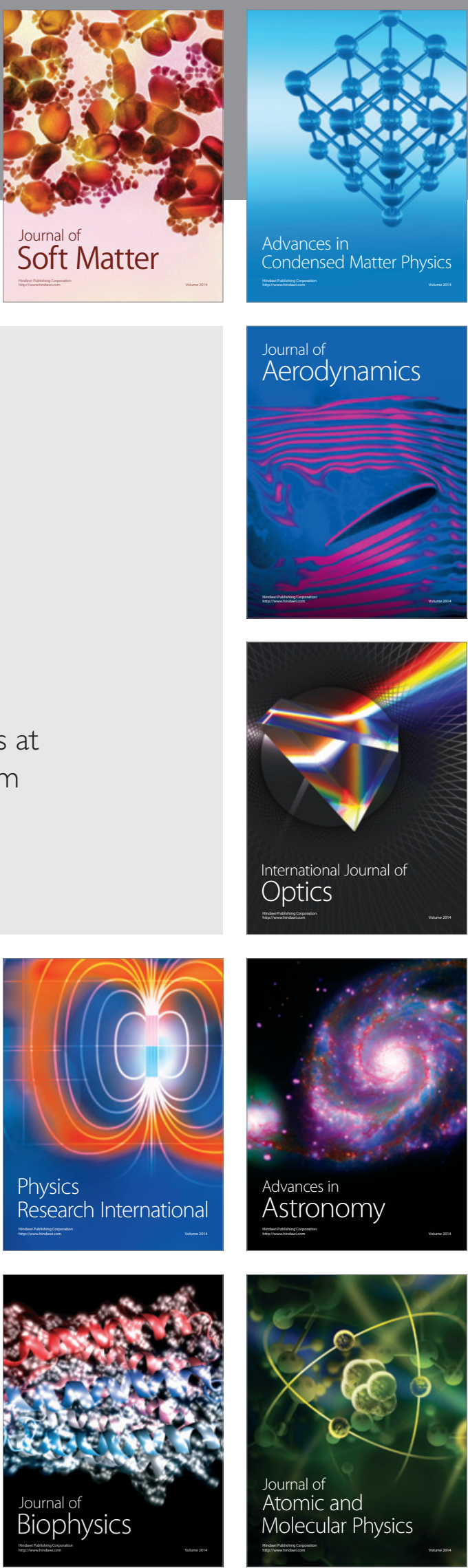